\title{
Circular RNA Expression in the Brain of a Neonatal Rat Model of Periventricular White Matter Damage
}

\author{
Lihua Zhu ${ }^{a}$ Ruibin Zhao ${ }^{b} \quad$ Li Huang $^{c}$ Sisi Moc Zhangbin Yu $^{d} \quad$ Li Jiang $^{c}$ \\ Lixing Qiaoc
}

Institute of clinical, Jiangsu Health Vocational College, Nanjing, bepartment of Pediatrics, Nanjing General Hospital, Nanjing, 'Department of pediatrics, Zhongda hospital, Southeast University, District, Nanjing, dDepartment of Pediatrics, Nanjing Maternity and Child Health Care Hospital, Nanjing Medical University, Nanjing, China

\section{Key Words}

Circular RNA • Deep RNA sequencing • Periventricular white matter damage • Premature birth - Hypoxia-ischemia

\begin{abstract}
Background/Aims: Periventricular white matter damage (PWMD) is the predominant neurologic lesion in preterm infants who survive brain injury. In this study, we assessed the global changes in and characteristics of the transcriptome of circular RNAs (circRNAs) in the brain tissues of rats with PWMD. Methods: We compared the expression profiles of circRNAs in brain samples from three rats with PWMD and three paired control tissues using deep RNA sequencing. Bioinformatics analysis was applied to investigate these differentially expressed circRNAs, and quantitative reverse-transcription polymerase chain reaction (qRT-PCR) analysis was performed to confirm the results. Gene Ontology (GO) and Kyoto Encyclopedia of Genes and Genomes (KEGG) analyses were performed to predict associated cell signaling pathways and functions. Network analysis was performed to predict circRNAs-microRNAs, and target genes related to PWMD. Results: A total of 2151 more reliable circRNAs were dysregulated in the brain tissues of rats with PWMD, indicating a potential role in the condition. Of the 98 circRNAs significantly differentially expressed in rat brains with PWMD $(P<0.05), 52$ were significantly over-expressed and 46 were significantly under-expressed. The expression profiles of seven of 10 randomly selected circRNAs were confirmed by qRT-PCR analysis. The glutamatergic synapse pathway and the VEGF signaling pathway, both associated with hypoxia/ischemia induced brain damage, were inriched. Relationship between miRNA (rnomiR-433-3p and rno-miR-206-3p) and HIF-1 $\alpha$ were evident and potential associations between chr6:48820833|48857932 and their target genes (rno-miR-433-3p and rno-miR-206-3p) were
\end{abstract}

L. Zhu and R. Zhao contributed equally to this work.

Li Jiang
and Lixing Qiao
Department of Pediatrics, Zhongda Hospital, Southeast University

Nanjing210009, Jiangsu (China)

Tel. +86 25 83205301, E-Mail jiangli77777@126.com; qiaolixing@aliyun.com 


\section{Cellular Physiology Cell Physiol Biochem 2018;49:2264-2276

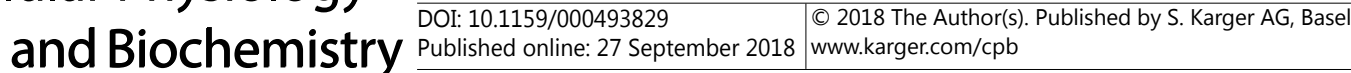 \\ Zhu et al.: Circular RNA and Periventricular White Matter Damage}

identified. Conclusion: The distinct expression patterns of circRNAs in the brain tissues of rats with PWMD suggest that circRNAs actively respond to hypoxia-ischemia. These findings could assist the development of novel diagnostic and therapeutic targets for PWMD therapy.

(C) 2018 The Author(s)

Published by S. Karger AG, Basel

\section{Introduction}

In premature infant, hypoxic-ischemic (HI) damage to cerebral white matter is a common and leading cause of brain injury that often results inchronic neurologic disability from cerebral palsy. During human brain development, between 23 and 32 weeks gestation, the periventricular cerebral white matter is at increased risk of injury from HI. HI, infection, inflammation, oxidative damage and excitotoxicity are pathogenic processes that collectively cause periventricular white matter damage (PWMD), and $\mathrm{HI}$ is believed to be a major factor in the evolution of PWMD [1].

Premature infants, particularly those with very low birth weight, brain injury are of enormous importance to public health because of the large numbers who survive the condition with serious neurodevelopmental disabilities, including major cognitive and behavioral defects $[2,3]$. PWMD is the predominant neurologic lesion in preterm infants who survive brain injury $[4,5]$. In China, approximately 1.5 million premature infants are born annually, among whom the incidence of PWMD is 5\% [6]. Moreover, the proportion of premature infants has gradually increased over the past decade. Thus, the incidence of neurologic disability caused by premature birth can be expected to rise. Therefore, the discovery of new diagnostic biomarkers and therapeutic targets with the potential to control and predict the prognosis of PWMD is of great importance.

Imaging is an important method of diagnosing PWMD in premature infants. Craniocerebral ultrasound is employed as a screening tool for PWMD, and demonstrates high sensitivity and specificity for brain damage. Magnetic resonance imaging has a high resolution and shows the complete skull structure, however, it takes a long time to check, which is unacceptable for severely ill children. The lack of standardization of imaging examinations contributes to variation in diagnostic results between clinics. In addition, many factors can influence the accuracy of sonograph investigations for PWMD, such as the experience of operators, quality of the equipment, lesion type, and departmental policies and guidelines at individual institutions. Extraordinary diagnostic precision leading to low morbidity and mortality characterize state of the art clinical management of PWMD. When caring for a neonate with PWMD, it is critical to determine whether there is an underlying genetic element associated with the disease. Recent advancements in molecular biology techniques have facilitated the elucidation of the molecular mechanisms underlying brain formation, which involve microRNAs (miRNAs) [7], long noncoding RNAs (lncRNAs) [8] and circular RNAs (circRNAs) [9]. However, little is known about the role of circRNAs in the pathology of PWMD.

Recently deep sequencing and advanced data analysis techniques have enabled the characterization of thousands of circRNAs in multiple tissues and organisms. CircRNAs are a large class of non-coding RNA that exist ubiquitously in the cytoplasm of eukaryotic cells $[10,11]$. In contrast to linear RNAs, circRNAs are single-stranded RNA molecules that form closed loops via covalent bonding [12] that have neither a 5'cap nor 3'tail, which may prevent degrdation by RNA exonuclease and thereby facilitate stable expression [13-15]. CircRNAs are mainly derive from the exonic regions of protein-coding genes, and are stable and varietyd in length. Endogenous circRNAs are thought to function as miRNA sponges, and are believed to antagonize miRNA-dependent gene regulation, thereby contributing to the competing endogenous RNA network [16]. In addition, circRNAs also regulate expressions of their parental genes. In Drosophila, circRNAs are most highly and specifically expressed in the brain [17]. This trend extends to mice and humans, suggesting that predominantly neural circRNA expression is conserved among species [13, 18, 19]. CircRNAs may have biologic roles relevant to the aging nervous system [20]. CircRNAs are widely involved in 
physiologic/pathologic processes, including nervous system disorders [9, 18, 21], cancer [22-24], and pre-eclampsia [25]. Boeckel et al [26]. found that endothelial circRNAs are regulated by hypoxia and have distinct biological functions. In a stroke mouse model, circRNAs are implicated in the pathological development of brain injury [27]. CircRNAs are gaining attention in investigation on the mechanisms underlying disease and on biomarkers for disease diagnosis and treatment.

To examine the roles of circRNAs in the pathogenesis of PWMD, we performed sequence analysis to identify circRNAs that are differentially expressed in the brain tissues of rats with white matter damage, compared with control animals. We aimed to generate laboratory data for future clinical studies to assist the development of circRNA as potential biomarkers and diagnostic tools for premature infants with PWMD.

\section{Materials and Methods}

\section{Animals}

Two Pregnant Sprague-Dawley (SD) rats (day 18-19 of the estrous cycle) were obtained from Nanjing Medical University of China and allowed to deliver. All animals were treated appropriately according to the Guide for the Care and Use of Laboratory Animals (National Research Council). Experimental processes were approved by the Southeast University Animal Experimentation Committee. Pups were housed with their mother under a constant $12 \mathrm{~h}$ dark $/ 12 \mathrm{~h}$ light cycle at $22^{\circ} \mathrm{C} \pm 2^{\circ} \mathrm{C}$ with free access to food and water.

\section{Neonatal Rat Model of Periventricular White Matter Damage}

As described in our previous study [28], postnatal day 3 (P3) rats underwent permanent ligation of the right common carotid artery after anesthetization with ether. Pups were returned to the home cage for $2 \mathrm{~h}$, then exposed to hypoxia ( $94 \% \mathrm{~N} 2+6 \% \mathrm{O} 2)$ for $2 \mathrm{~h}$ by being placed in a sealed chamber partially submersed in a $37^{\circ} \mathrm{C}$ water bath. At the end of the hypoxia treatment, pups were returned to their dam for recovery. In control rats, the same surgery was performed without ligation and exposure to hypoxia.

\section{Brain Tissue Preparation}

Rats were sacrificed with a lethal dose of pentobarbital ( $>50 \mathrm{mg} / \mathrm{kg}$ i.p.) at $24 \mathrm{~h}$ after operation. For hematoxylin and eosin (HE) staining (three control rats and three $\mathrm{HI}$ rats), brain tissues (3mm either side of the optic chiasm in the coronal plane) were removed after decapitation and fixed in $4 \%$ paraformaldehyde overnight at $4^{\circ} \mathrm{C}$. For cryoprotection, brain tissue was transferred sequentially to $30 \%$ sucrose in $0.1 \mathrm{MPBS}$ until it sank. Then embedded in O.C.T (Zhongshan Biotechnology Co., Ltd., Beijing, China) and stored at $-80^{\circ} \mathrm{C}$. Three control rats and three $\mathrm{HI}$ rats were used for sequencing, and six control rats and six HI rats were used for quantitative reverse-transcription polymerase chain reaction (qRT-PCR), the right brains were rapidly harvested, snap frozen in liquid nitrogen and kept at $-80^{\circ} \mathrm{C}$.

Fig. 1. $\quad \mathrm{HE}$ stained coronal brain sections. (A) The white matter under the cortex and corpus callosum appeared normal in sham rats. (B) In rats with PWMD, the right

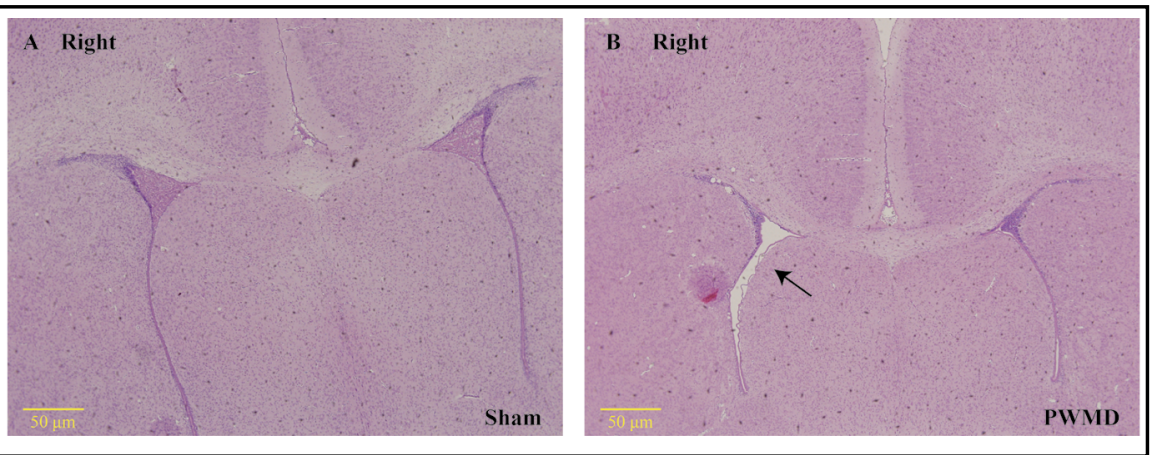

white matter exhibited leukoaraiosis, and the right ventricle appeared expanded (arrow). 


\section{Cellular Physiology Cell Physiol Biochem 2018;49:2264-2276 and Biochemistry \begin{tabular}{l|l} 
DOI: 10.1159/000493829 & (c) 2018 The Author(s). Published by S. Karger AG, Basel \\
www.karger.com/cpb
\end{tabular}

\section{Hematoxylin and Eosin (HE) Staining}

Consecutive frozen brain sections were used for HE staining. The HE stained sections were examined under the microscope for any alteration in histopathology. Slides were examined with a computer-assisted Olympus $\mathrm{CK}_{2}$ microscope. Five sections from each rat were taken from the right white matter region. In the right brain of $\mathrm{HI}$ rats, the white matter under the cortex and corpus callosum exhibited serious leukoaraiosis, ventricles were expanded, the arrangement of neurons in the gray matter was disordered, and apoptosis was evident (Fig. 1).

\section{Whole Transcriptome Library Preparation}

Total RNA was obtained using TRIzol (Invitrogen, UK) according to the manufacturer's protocol. Total genomic DNA was then removed from RNA samples using DNase I (NewEngland Biolabs) and RNA purity was assessed using a Nanodrop-2000 instrument. Each RNA sample had an A260:A280 ratio above 1.9 and an A260:A230 ratio above 1.8. Total RNA was subject to ribosomal RNA depletion according to the manufacturer's protocol supplied with the Ribo-Minus kit (Life Technology). Next, RNA was fragmented into 200 300 base pairs (bp) using an RNA fragmentation kit (Ambion) and quantified with Nanodrop instrument (Thermo Scientific). The first cDNA strand was synthesized using random hexamer primers, and the second strand cDNA was synthesized using dUTP instead of dTTP. In this step, actinomycin D was used to increase strand specificity by inhibiting second-strand cDNA synthesis. At $15^{\circ} \mathrm{C} 0.5 \mu \mathrm{l}$ of actinomycin D solution $(120 \mathrm{ng} / \mu \mathrm{l}$ ), $0.5 \mu \mathrm{l}$ of RNase OUT (40 units/ $\mu$ l, Invitrogen) and $0.5 \mu \mathrm{l}$ of SuperScript III polymerase (200 units/ $\mu \mathrm{l}$, Invitrogen) were added to the reaction. Then, EB $(20 \mu \mathrm{l})(10 \mathrm{mM}$ Tris-Cl, pH 8.5, Qiagen) was

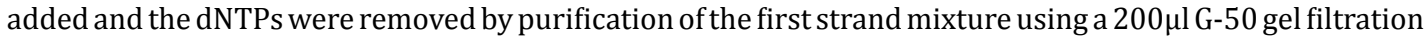
spin-column equilibrated with $1 \mathrm{mM}$ Tris-Cl, $\mathrm{pH}$ 7.0. After second strand synthesis and DNA fragmentation, sequencing libraries were constructed by following the manufacturer's instructions (Illumina). Fragments of 300-400 bp were recovered and purified, then enriched by 15 cycles of PCR. Each library was loaded into one lane of an Illumina HiSeq 2500 for $2 \times 150$ bp pair-end (PE) sequencing.

\section{Deep RNA sequencing (RNA-seq)}

We used FastQC (http://www.bioinformatics.babraham.ac.uk/projectissue specific/fastqc/) to control the quality of the sequencing data. At an initial filtering step, we discarded low quality reads, including reads that had adaptors, reads with $\geq 10 \%$ unknown bases, and reads that had $\geq 50 \%$ bases with a quality value $\leq 5$.

Next, we applied CIRI [29] to identify circRNAs. For the CIRI algorithm, we first mapped the filtered data to the Rattus_norvegicus.Rnor_6.0 genome reference using BWA [30] software. Based on the Sequence Alignment Map file, CIRI was used to detect circRNAs.

To ensure the accuracy of circRNA identification, we retained only high-confidence circRNAs by adopting two criteria: selecting circRNAs identified in at least two junction reads; and selecting circRNAs present in more than four samples.

To obtain the full-length nucleotide sequence of all circRNA isoforms, we compared the back-spliced junction sites with the Rattus_norvegicus.Rnor_6.0 genome annotation downloaded from UCSC [31] database. Reportedly, multiple circRNA isoforms can be generated from the same back-spliced junction site $[32,33]$. Based on the method of Liu et al [34]., we identified multiple circRNA isoforms with annotated transcripts if the "head" and "tail" positions of the detected back-spliced junction were located exactly at exon junction sites or if part of the junction sites had small flanking portions of intron sequencing. We also identified some circRNA isoforms associated with their back-spliced junction sites located in intergenic regions, with no overlap with known genes or overlap with known genes but localized on their antisense strands. CircRNAs that were fully located in intronic regions were also retained. CircRNAs obtained from each sample were filtered (standard: junction reads $\geq 2$ ), and all circRNAs present in more than four samples were collated. To assess differentially expressed circRNAs, raw counts of reads spanning a particular headto-tail junction were analyzed using edgeR package (version 3.12.1) with TMM normalization in generalized linear model. The Trimmed Mean of M-values (TMM) normalization method was used to minimize size bias between samples for sequencing libraries. Significantly differentially expressed circRNAs were those exhibiting a fold change $(\mathrm{FC}) \geq 1.5$ and $\mathrm{P}$-value $<0.05$. All statistical analyses were performed using R 3.2.3 (http://www.r-project.org/).

\section{KARGER}




\section{Cellular Physiology Cell Physiol Biochem 2018;49:2264-2276 and Biochemistry Published \begin{tabular}{l|l} 
DOI: 10.1159/000493829 & (c) 2018 The Author(s). Published by S. Karger AG, Basel \\
www.karger.com/cpb
\end{tabular} \\ Zhu et al.: Circular RNA and Periventricular White Matter Damage}

Confirmation of CircRNA Expression via Quantitative Real-Time Polymerase Chain Reaction (qRT-PCR) Analysis

Ten circRNAs we selected for qRT -PCR analysis based on the following conditions; (1) statistically significant differences between control and PWMD groups in the RNA-Seq analysis; (2) relatively high expression; (3) the host gene was normal in HI-induced brain damage. Total RNA was isolated from neonatal rat brains using TRIzol reagent (Invitrogen, UK) according to the manufacturer's protocol. The purity and concentration of RNA were evaluated by measuring the OD260/280 ratio in a spectrophotometer. Total RNA was reverse transcribed using a PrimeScript ${ }^{\mathrm{TM}} \mathrm{RT}$ reagent kit (Takara, Otsu, Shiga, Japan). After cDNA synthesis, real-time PCR was performed using SYBR Premix Ex Taq ${ }^{\mathrm{TM}}$ (Takara) for amplification of the PCR products. The $\beta$-actin gene was chosen as a reference gene. All reactions were performed in triplicate with the same cDNA samples. The final results were expressed as the relative expression ratio between the targeted gene and the $\beta$-actin reference gene. Relative gene expression was analyzed using the $2^{-\Delta \Delta \mathrm{Ct}}$ method. Primer information is listed in Table 1.

\section{Bioinformatics analysis}

We performed Gene Ontology (GO) and Kyoto Encyclopedia of Genes and Genomes (KEGG) enrichment analyses of the parent genes of all specific differentially expressed circRNAs, and removed duplicate genes using DAVID v6.7 $[35,36]$. KEGG pathway enrichment analysis was performed using the KEGG Orthology Based Annotation System (KOBAS 3.0, http://kobas.cbi.pku.edu.cn/anno_iden.php) [37].

We selected specific circRNAs and construct a network according to the circRNA profiling data. CircRNA-miRNA interaction were predicted using Arraystar miRNA target prediction software based on TargetScan and miRanda [27, 38], and miRNA-mRNA interactions were predicted using miRTarBase [27].

\section{Statistical Analysis}

All data in this article are expressed as means \pm standard deviation. Graphs were generated using Microsoft Excel (Microsoft Corp., Redmond,WA, USA). Statistical analysis was performed using SPSS 18.0 (SPSS Inc., Chicago,IL, USA). Differences between the two groups were determined using the independentsamples t-test. Probability levels of 0.05 were considered statistically significant.

\section{Results}

\section{Results of Deep RNA Sequencing}

We predicted 2151 probable circRNAs. Based on the circRNA expression profiles, differentially expressed circRNAs were identified in the PWMD group compared with the sham group. Hierarchical clustering was performed to group circRNAs based on their expression levels (Fig. 2A). We set a threshold of a fold change $>1.5$ and a p-value $<0.05$. We found that 98 circRNAs were differentially expressed in the PWMD group (Fig. 2B and 2C), of which the top 40 are listed in Table 2 . Of them, 52 were significantly over-expressed and 46 were significantly under-expressed. Among the up-regulated circRNAs, 13 were intergenic, one was intronic, and 38 were exonic. Among the down-regulated circRNAs, seven were intergenic, one was intronic, and 38 were exonic (Fig. 2D). 


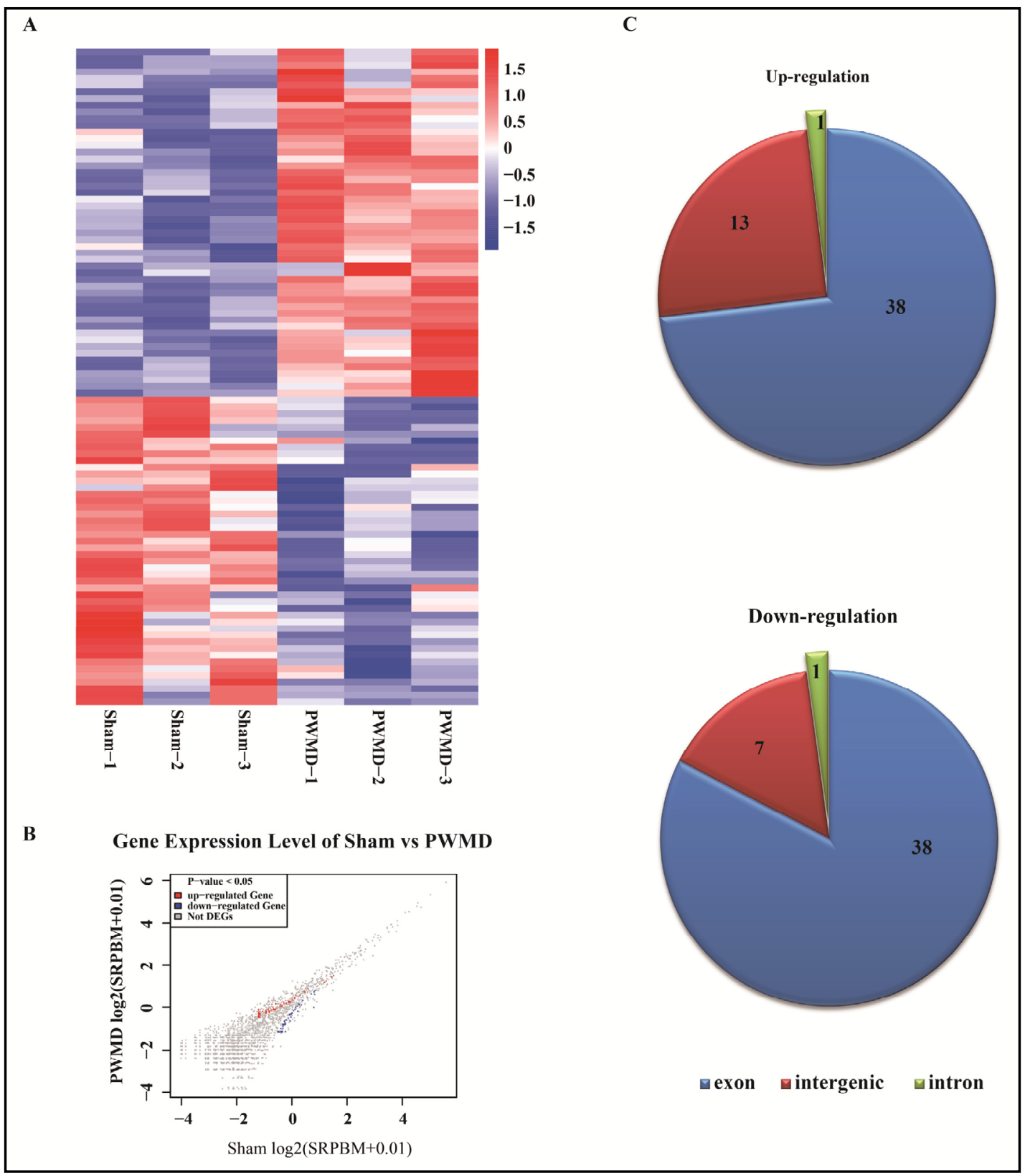

Fig. 2. Analysis of differentially expressed circRNAs. (A) The hierarchical clustering of partial differentially expressed circRNAs. 'Red' indicates high relative expression, and 'blue' indicates low relative expression. (B) CircRNAs in the Scatter-Plot. 'Red' indicates up-regulation and 'blue' indicates down-regulation more than 1.5 fold change of circRNAs between the two groups. (C) Classification of dysregulated 1.5-fold up and down expressed circRNAs with statistical significance $(\mathrm{P}<0.05)$.

Validation of CircRNA Expression

We randomly selected 10 circRNAs, including four up-regulated circRNAs (chr1:17536371|17554677, chr2:41261840|41286354, chr15:837322|852690, and chr14:9421418|9440942) and six down-regulated circRNAs (chr18:24611733|24614740, chr18:73479863|73498648, chr7:114487838|114499158, chr7:30727478|30735182, chr6:48820833|48857932, and chr16:63915078|63933482) for verification by qRT-PCR analysis. A general consistency was evident between the real-time PCR and RNA-seq results. The expression of two of the selected up-regulated circRNAs (chr1:17536371|17554677

\section{KARGER}


and chr15:837322/852690), five of the selected down-regulated circRNAs (chr18:24611733|24614740, chr7:114487838|114499158, chr6:48820833|48857932 and chr16:63915078|63933482, $P<0.01$ ) (chr18:73479863|73498648, $P<0.05$ ) were confirmed (Fig. 3). There wasere no significant differences for chr7:30727478|30735182 $(P=0.31)$ or chr14:9421418|9440942 $(P=0.619)$ between two groups by qRT-PCR analysis. However, the expression trends of both were consistent with those determined using RNAseq. The expression trend of chr2:41261840|41286354 was not confirmed.

\section{Gene Ontology and KEGG} Enrichment Analysis

Under the assumption that the function of circRNAs is related to the known function of the host genes, we performed GO enrichment and KEGG pathway analyses to predict the potential functions of deregulated circRNAs.

GO enrichment analysis suggested that the host genes of PWMD specific circRNAs were of several functional categories. Highly enriched GO terms associated with circRNA transcripts among biological process, cellular components, and molecular functions are shown in Table 3. KEGG enrichment analysis

Table 2. Top 40 differentially expressed circRNAs between PWMD and sham groups. FDR, false discover rate; FC, fold change. The circular RNAs identified to be dysregulation by $\mathrm{P}$ value $<0.05$

\begin{tabular}{|c|c|c|c|c|c|c|}
\hline circRNA & P-value & FDR & FC & Regulation & circRNA type & Gene Symbol \\
\hline chr1:17536371|17554677 & 0.000 & 0.062 & 9.879 & up & exon & Ptprk \\
\hline chr14:86326421|86326743 & 0.001 & 0.347 & 7.513 & up & exon & Nudcd3 \\
\hline chr3:29584278|29597359 & 0.006 & 0.630 & 6.713 & up & intron & Gtdc1 \\
\hline chr15:837322|852690 & 0.015 & 0.782 & 6.428 & up & exon & Kcnma1 \\
\hline chr9:50892486|50906966 & 0.016 & 0.782 & 6.379 & up & intergenic & \\
\hline chr6:14450092|14758647 & 0.015 & 0.782 & 6.331 & up & intergenic & - \\
\hline chr6:30550488|30589369 & 0.001 & 0.352 & 6.297 & up & intergenic & - \\
\hline chr7:126265888|126283984 & 0.014 & 0.782 & 6.003 & up & exon & Atxn10 \\
\hline chr5:94693114|95108520 & 0.015 & 0.782 & 5.939 & up & intergenic & \\
\hline chr14:9421418|9440942 & 0.024 & 0.903 & 5.619 & up & exon & Cds1 \\
\hline chr4:34740521|34761519 & 0.038 & 0.963 & 5.607 & up & exon & Ica1 \\
\hline chr6:7718824|7738462 & 0.006 & 0.630 & 5.600 & up & exon & Thada \\
\hline chr1:241731334|241732616 & 0.047 & 0.963 & 5.598 & up & exon & Apba1 \\
\hline chr1:175200414|175218889 & 0.020 & 0.889 & 5.221 & up & exon & Sbf2 \\
\hline chr1:157470071|157490006 & 0.046 & 0.963 & 4.860 & up & exon & Pcf11 \\
\hline chr19:38057557|38062193 & 0.005 & 0.610 & 4.690 & up & exon & Nfatc3 \\
\hline chr2:116586880|116594945 & 0.000 & 0.126 & 4.620 & up & intergenic & . \\
\hline chr1:119172338|119555633 & 0.011 & 0.761 & 4.542 & up & intergenic & - \\
\hline chr16:39881896|39884207 & 0.047 & 0.963 & 4.454 & up & exon & Wdr17 \\
\hline chr3:92183759|92225505 & 0.047 & 0.963 & 4.453 & up & exon & Trim44 \\
\hline chr18:24611733|24614740 & 0.000 & 0.126 & 9.786 & down & exon & Wdr33 \\
\hline chr11:83225853|83225993 & 0.002 & 0.359 & 8.241 & down & intron & Vps8 \\
\hline chr12:39627016|39635912 & 0.003 & 0.466 & 7.435 & down & exon & Anapc7 \\
\hline chr14:28418126|28465972 & 0.008 & 0.722 & 7.384 & down & exon & Adgrl3 \\
\hline chr7:114487838|114499158 & 0.009 & 0.734 & 7.062 & down & exon & Ptk2 \\
\hline chr18:73479863|73498648 & 0.009 & 0.722 & 6.557 & down & exon & Pias2 \\
\hline chr9:118986481|118991497 & 0.009 & 0.722 & 6.543 & down & exon & Dlgap1 \\
\hline chr13:97559165|97566714 & 0.040 & 0.963 & 5.632 & down & intergenic & - \\
\hline chr10:74338952|74360926 & 0.008 & 0.722 & 5.538 & down & exon & Gdpd1 \\
\hline chr18:13369444|13417002 & 0.044 & 0.963 & 5.180 & down & intergenic & \\
\hline chr4:182811463|182826795 & 0.045 & 0.963 & 5.168 & down & exon & Tmtc1 \\
\hline chr16:54054981|54061837 & 0.046 & 0.963 & 5.156 & down & exon & Pcm1 \\
\hline chr3:15042229|15042609 & 0.041 & 0.963 & 4.650 & down & exon & Dab2ip \\
\hline chr3:21850499|21878612 & 0.001 & 0.279 & 4.644 & down & exon & Strbp \\
\hline chr2:195751903|195795666 & 0.044 & 0.963 & 4.623 & down & exon & Snx27 \\
\hline chr18:5191058|5194413 & 0.034 & 0.963 & 4.382 & down & exon & Zfp521 \\
\hline chr7:124682871|124727275 & 0.004 & 0.527 & 4.314 & down & exon & Mpped1 \\
\hline chr15:34277381|34277914 & 0.013 & 0.782 & 4.140 & down & exon & Rnf31 \\
\hline chr1:195625166|195866944 & 0.039 & 0.963 & 3.956 & down & intergenic & - \\
\hline chr14:81163989|81182711 & 0.024 & 0.903 & 3.730 & down & exon & $\mathrm{Htt}$ \\
\hline
\end{tabular}

Fig. 3. Validation of ten circRNAs expression by qRTPCR. The values shown are the means \pm standard deviation. ** means $\mathrm{P}<0.01$ vs. the sham group, * means $\mathrm{P}<0.05$ vs. the sham group, and the expressions were consistent between sequencing and realtime PCR. \# means $\mathrm{P}<0.01$ vs. the sham group, but the expressions were inconsistent between sequencing and realtime PCR. No sign means of $\mathrm{P}>0.05$ vs. the sham group, however consistent with the trend of sequencing.

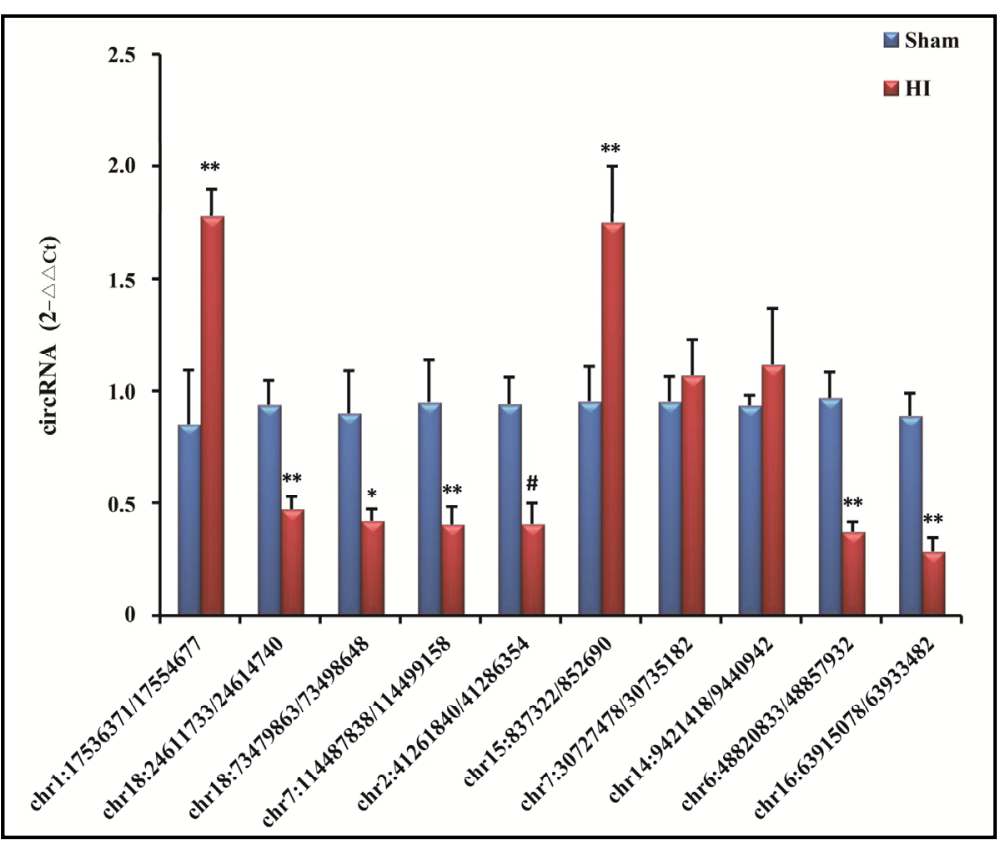


revealed that the host genes of circRNAs were enriched in some PWMD function related pathways. One of the most enriched signaling pathways was the glutamatergic synapse pathway, and the vascular endothelial growth factor (VEGF) signaling pathway was also enriched (Table 4). Interesting, both of these pathway arereportedly involved in $\mathrm{HI}$ induced brain damage $[39,40]$.

Prediction of CircRNAs/ miRNAs and Target Genes

Recent studies have reported that circRNAs can function as miRNA sponges to modulate miRNA expression and miRNA-dependent gene regulation $[13,16,41]$. This interaction between circRNAs and diseaseassociated miRNAs suggests that circRNAs function in disease regulation $[16,21$, 41]. Two confirmed circRNAs (chr6:48820833|48857932 a $\quad \mathrm{n}$ d chr16:63915078|63933482) and two circRNAs (chr7:30727478|30735182 and chr14:9421418|9440942) for which the expression trends were consistent with RNA-seq data were annotated in detail using circRNA-miRNA interaction information. The potential miRNA targets of circRNAs and mRNA targets of miRNA list in Supplementary Fig. S1 (For all supplementary material see www.karger. com/10.1159/000493829/). Most of the identified circRNAs contain one or more miRNA binding sites, and some appear to bind tightly to one miRNA via multiple sites. For example, rno-miR-216a$5 p$ is strongly complementary to three circRNAs
Table 3. Significantly enriched GO terms for differentially expressed circRNA genes between PWMD and sham groups

\begin{tabular}{|c|c|c|c|}
\hline Category & Name & Count & P-value \\
\hline Molecular-function & protein binding & 18 & 0.000 \\
\hline Molecular-function & protein domain specific binding & 8 & 0.000 \\
\hline Molecular-function & phosphatidylinositol 3-kinase binding & 3 & 0.004 \\
\hline Molecular-function & zinc ion binding & 11 & 0.005 \\
\hline Molecular-function & identical protein binding & 8 & 0.010 \\
\hline Molecular-function & ion channel binding & 4 & 0.012 \\
\hline Molecular-function & protein complex binding & 6 & 0.012 \\
\hline Molecular-function & protein kinase binding & 6 & 0.024 \\
\hline Molecular-function & metal ion binding & 11 & 0.033 \\
\hline Celluar-component & cytosol & 19 & 0.000 \\
\hline Celluar-component & postsynaptic density & 7 & 0.000 \\
\hline Cellular-component & axon & 8 & 0.000 \\
\hline Cellular-component & dendrite & 9 & 0.000 \\
\hline Cellular-component & synapse & 7 & 0.000 \\
\hline Celluar-component & neuronal cell body & 9 & 0.000 \\
\hline Cellular-component & cytoplasm & 32 & 0.002 \\
\hline Cellular-component & cell junction & 6 & 0.018 \\
\hline Celluar-component & nucleoplasm & 13 & 0.026 \\
\hline Cellular-component & membrane & 15 & 0.030 \\
\hline Celluar-component & postsynaptic membrane & 4 & 0.037 \\
\hline Biological-process & brain development & 7 & 0.000 \\
\hline Biological-process & regulation of GTPase activity & 4 & 0.001 \\
\hline Biological-process & neuron projection development & 5 & 0.002 \\
\hline Biological-process & intracellular protein transport & 5 & 0.007 \\
\hline Biological-process & positive regulation of neuron projection development & 4 & 0.015 \\
\hline Biological-process & positive regulation of protein kinase activity & 3 & 0.020 \\
\hline Biological-process & regulation of ion homeostasis & 2 & 0.022 \\
\hline Biological-process & positive regulation of phosphatidylinositol 3-kinase signaling & 3 & 0.023 \\
\hline Biological-process & positive regulation of ion transmembrane transporter activity & 2 & 0.025 \\
\hline Biological-process & chemical synaptic transmission & 4 & 0.026 \\
\hline Biological-process & protein autophosphorylation & 4 & 0.031 \\
\hline Biological-process & positive regulation of axon regeneration & 2 & 0.036 \\
\hline Biological-process & nervous system development & 4 & 0.037 \\
\hline Biological-process & positive regulation of protein kinase B signaling & 3 & 0.039 \\
\hline
\end{tabular}

Table 4. KEGG Pathway for differentially expressed circRNAs between PWMD and sham groups

\begin{tabular}{|c|c|c|c|}
\hline Term & ID & P-value & Input gene \\
\hline Glutamatergic synapse & rno04724 & 0.000 & Prkcb;Grik2;Ppp3cc;Dlgap1 \\
\hline VEGF signaling pathway & rno04370 & 0.001 & Prkcb;Ppp3cc;Ptk2 \\
\hline Ubiquitin mediated proteolysis & rno04120 & 0.001 & Pias2;Anapc7;Trip12;Herc1 \\
\hline ErbB signaling pathway & rno04012 & 0.004 & Prkcb;Ptk2;Nrg \\
\hline Ras signaling pathway & rno04014 & 0.009 & Prkcb;Tiam1;Ksr2;Igf1r \\
\hline Amino sugar and nucleotide sugar metabolism & rno00520 & 0.013 & Uxs1;Cyb5r4 \\
\hline Wnt signaling pathway & rno04310 & 0.015 & Prkcb;Nfatc3;Ppp3cc \\
\hline Long-term depression & rno04730 & 0.020 & Prkcb;Igf 1r \\
\hline Long-term potentiation & rno04720 & 0.021 & Prkcb;Ppp3cc \\
\hline cGMP-PKG signaling pathway & rno04022 & 0.021 & Kcnma1;Nfatc3;Ppp3cc \\
\hline Axon guidance & rno04360 & 0.026 & Ptk2;Nfatc3;Ppp3cc \\
\hline Chemokine signaling pathway & rno04062 & 0.026 & Prkcb;Tiam1;Ptk2 \\
\hline Focal adhesion & rno04510 & 0.034 & Prkcb;Ptk2;Igf1r \\
\hline Rap1 signaling pathway & rno04015 & 0.039 & Prkcb;Tiam1;Igf1r \\
\hline Regulation of actin cytoskeleton & rno04810 & 0.040 & Tiam1;Ptk2;Iqgap2 \\
\hline Phosphatidylinositol signaling system & rno04070 & 0.046 & Prkcb;Cds1 \\
\hline Natural killer cell mediated cytotoxicity & rno04650 & 0.047 & Prkcb;Ppp3cc \\
\hline Retrograde endocannabinoid signaling & rno04723 & 0.054 & Prkcb;Rims1 \\
\hline HIF-1 signaling pathway & rno04066 & 0.055 & Prkcb;Igf 1r \\
\hline $\mathrm{T}$ cell receptor signaling pathway & rno04660 & 0.059 & Nfatc3;Ppp3cc \\
\hline MAPK signaling pathway & rno04010 & 0.065 & Prkcb;Nfatc3;Ppp3cc \\
\hline Vascular smooth muscle contraction & rno04270 & 0.066 & Prkcb;Kcnma1 \\
\hline Dopaminergic synapse & rno04728 & 0.077 & Prkcb;Ppp3cc \\
\hline mTOR signaling pathway & rno04150 & 0.111 & Prkcb;Igf1r \\
\hline Calcium signaling pathway & rno04020 & 0.131 & Prkcb;Ppp3cc \\
\hline Carbohydrate digestion and absorption & rno04973 & 0.135 & Prkcb \\
\hline cAMP signaling pathway & rno04024 & 0.153 & Tiam1;Pde4d \\
\hline Synaptic vesicle cycle & rno04721 & 0.193 & Rims1 \\
\hline GABAergic synapse & rno04727 & 0.277 & Prkcb \\
\hline NF-kappa B signaling pathway & rno04064 & 0.280 & Prkcb \\
\hline PI3K-Akt signaling pathway & rno04151 & 0.321 & Ptk2;Igf1r \\
\hline Cholinergic synapse & rno04725 & 0.326 & Prkcb \\
\hline Serotonergic synapse & rno04726 & 0.353 & Prkcb \\
\hline Sphingolipid signaling pathway & rno04071 & 0.357 & Prkcb \\
\hline AMPK signaling pathway & rno04152 & 0.367 & Igf1 $\mathrm{r}$ \\
\hline FoxO signaling pathway & rno04068 & 0.369 & Igf1r \\
\hline Apoptosis & rno04210 & 0.383 & Dab2ip \\
\hline Tight junction & rno04530 & 0.389 & Prkcb \\
\hline Jak-STAT signaling pathway & rno04630 & 0.418 & Pias2 \\
\hline Protein processing in endoplasmic reticulum & rno04141 & 0.443 & Ubqln1 \\
\hline Alzheimer's disease & rno05010 & 0.451 & Рpp3cc \\
\hline Purine metabolism & rno00230 & 0.469 & Pde4d \\
\hline Endocytosis & rno04144 & 0.620 & Igf1r \\
\hline Neuroactive ligand-receptor interaction & rno04080 & 0.634 & Grik2 \\
\hline
\end{tabular}




\section{Cellular Physiology Cell Physiol Biochem 2018;49:2264-2276

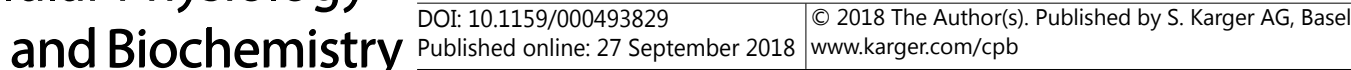

(chr7:30727478|30735182, chr6:48820833|48857932 and chr16:63915078|63933482). We then examined the mRNA binding results and found that chr6:48820833|48857932 and chr16:63915078|63933482 can bind to the miRNA effector pten. We also identified potential associations between chr6:48820833|48857932 and their target genes (rno-miR433-3p and rno-miR-206-3p), and potential relationships between miRNA rno-miR-433-3p and HIF- $1 \alpha$, rno-miR-206-3p, and HIF- $1 \alpha$.

\section{Discussion}

In this study, we examined the transcriptome of circRNAs in a neonatal rat model of PWMD. This study is the first to profile circRNAs differentially expressed in rats with PWMD compared with healthy rats.

CircRNAs are non-coding RNAs (ncRNAs) produced in eukaryotic cells during posttranscriptional processes. Recent studies have revealed that circRNAs are primarily generated from exons or introns of their parental genes [13, 42, 43] and can regulate the expression of parental genes [44-46]. Many exonic transcripts can form circRNAs through non-linear reverse splicing or gene rearrangement. CircRNAs can potentially regulate multiple aspects of cellular physiology, including miRNA binding, translational regulation, protein interactions, and even protein translation. RNA-seq of transcripts showed that, in the brain, a significantly greater fraction of reads are circRNA junction reads, a greater number of genes are hosts to circRNAs, and there are many tissue-specific circRNA hosts [47]. CircRNAs contain highly conserved sequences and display a high degree of stability in mammalian cells [18]. These properties mean that circRNAs have the potential to be used as stable biomarkers and potential therapeutic targets [48].

In this study, we chose RNA-seq to identify circRNAs. Microarray analysis is the most popular technology for the investigation of global RNA expression, involving probes designed to recognize annotated genes or sequences present in the genome. However, microarray analysis cannot detect undiscovered circRNAs. By contrast, RNA-seq can, in principle, detect any expressed RNAs and identify "aberrant" junction sequences, thereby signaling the presence of circRNAs [49]. In this study, we predicted 2151 probable circRNAs in silico, and selected circRNAs that co-occurred in more than four samples for further analysis. Thus, the true number of circRNAs is certainly much larger.

A recent evaluation of five different circRNA prediction algorithms found dramatic differences between their outputs, emphasizing that these prediction results should be handled with care [50]. Therefore, for any candidate circRNA identified, further independent experimental validation is required [51]. In this study, we validated the expression of 10 circRNAs using qRT-PCR. As a result, the expression of two up-regulated circRNAs and five down-regulated circRNAs was confirmed. Although there was no significant difference in the expression of circRNAs chr7:30727478|30735182 and chr14:9421418|9440942 between the two groups according to qRT-PCR analysis, their expression trends were consistent with those detected by RNA-seq. Increasing the number of samples may yield clearer results. However, this validity is consistent with that of other studies [52]. At the time of writing, these circular RNAs had not been identified in other studies using similar samples.

To investigate the functions of these PWMD specific circRNAs, we performed GO enrichment and KEGG pathway analyses of their annotated host protein coding genes. GO enrichment analyses revealed that some genes were involved in the regulation of biological processes, cellular components, and molecular functions. Signaling pathways such as the glutamatergic synapse pathway and VEGF pathway are reportedly associated with HI induced brain injury. Liu et al [39]. found that the thalamocortical circuitry is affected and vulnerable in PWMD mice and glutamatergic synaptic transmission is associated with such changes. Meanwhile, VEGF signaling pathway is involved in anoxia in rats, and recombinant human erythropoietin promotes brain recovery through this pathway [40]. These results showed that these genes generate one or more specifically expressed circRNAs, suggesting 


\section{Cellular Physiology Cell Physiol Biochem 2018;49:2264-2276

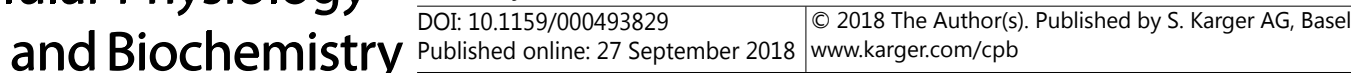 \\ Zhu et al.: Circular RNA and Periventricular White Matter Damage}

that these PWMD specific circRNAs play important roles in the molecular basis of phenotype heterogeneity in neonates with PWMD.

CircRNAs can function as a miRNA sponges, and may alleviate the inhibitory effects of miRNAs on target molecules, thereby regulating gene expression. Reportedly, the antisense sequence of cerebellar degeneration-related protein1 transcript (CDR1as) is a representative molecular sponge. CDR1as contains approximately 74 binding sites for miR7 , and CDR1as over-expression down-regulates miR-7 expression [18, 53]. In this study, we identified many dysregulated circRNAs in the brains of rats with PWMD. We found that most of the circRNAs contained one or more miRNA binding sites. We found that some circRNAs could bind densely to one miRNA. Therefore, we deduced that circRNAs probably compete with other RNAs for miRNA binding. HIF- $1 \alpha$ is a member of the Hypoxia Inducible Factor (HIF) family. Endogenous hypoxia-inducible mechanisms are crucial during the early stages of brain development. Hypoxia appears to activate mitogen-activated protein kinase that phosphorylates HIF- $1 \alpha$ and thereby stabilizes the molecule. In the neonatal brain, when hypoxia is accompanied by ischemia there are more persistent alterations of HIF- $1 \alpha$ expression. To date, more than 100 target genes of HIF-1 $\alpha$ have been identified with varying functions, including erythropoiesis/iron metabolism, angiogenesis, vascular tone, matrix metabolism, glucose metabolism, cell proliferation/survival, necrosis, anti-apoptosis and apoptosis [54]. We found that HIF-1 $\alpha$ was significantly altered in the brain tissue of neonatal rats after hypoxic ischemia [55]. This network indicated a targeted relationship between miRNA (rno-miR-433-3p and rno-miR-206-3p) and HIF- $1 \alpha$, and potential associations between chr6:48820833|48857932 and their target genes (rno-miR-433-3p and rno-miR206-3p). This may represent one of the key mechanisms in PWMD. To date, a clear function has only been demonstrated for a few circRNAs that serve as miRNA sponges $[13,16,56]$, sequestering miRNAs and preventing their interactions with target mRNAs. Further analysis is warranted to prove that these circRNAs act as a miRNA sponges.

There are some limitations of the present study. First, translating these findings to humans may be difficult due to species-specific expression pattern of circRNAs. In future studies, we will use a human in vitro model of hypoxia to verify that the dysregulation of candidate circRNAs is conserved between species. Second, translating the findings to the clinic may prove problematic due to the lack of rapid and easy detection of circRNAs associated with PWMD. Thus, we will obtain information on the expression profiles of circRNAs from the plasma and serum of PWMD and control rats and premature infants, and select circRNAs for functional and mechanic studies.

\section{Conclusion}

We showed that circRNAs are dysregulated in the brains of rats with PWMD compared with sham rats. These findings may provide preliminary data that can be used to identify candidate for PWMD diagnosis, and insight into the mechanisms of PWMD.

\section{Acknowledgements}

This project was supported by grants from the National Natural Science Foundation of China (nos. 81601355 and 81771628) and the Natural Science Foundation of Jiangsu Health Vocational College (JKA201705).

\section{Disclosure Statement}

The authors have no conflicts of interest to disclose. The authors have no financial relationships relevant to this article to disclose. 


\section{Cellular Physiology Cell Physiol Biochem 2018;49:2264-2276 \begin{tabular}{ll|l} 
and Biochemistry Published online: 27 September 2018 & $\begin{array}{l}\text { (c) } 2018 \text { The Author(s). Published by S. Karger AG, Basel } \\
\text { www.karger.com/cpb }\end{array}$
\end{tabular}

\section{References}

1 Volpe JJ: Neurology of the Newborn, ed 4. Philadelphia: WB Saunders, 2001.

$\rightarrow 2$ Johnson S, Marlow N: Positive screening results on the modified checklist for autism in toddlers: Implications for very preterm populations. J Pediatr 2009;154:478-480.

3 Larroque B, Ancel PY, Marret S, Marchand L, Andre M, Arnaud C, Pierrat V, Roze JC, Messer J, Thiriez G, Burguet A, Picaud JC, Breart G, Kaminski M: Neurodevelopmental disabilities and special care of 5-yearold children born before 33 weeks of gestation (the EPIPAGE study): A longitudinal cohort study. Lancet 2008;371:813-820.

4 Liu XB, Shen Y, Plane JM, Deng WB: Vulnerability of premyelinating oligodendrocytes to white-matter damage in neonatal brain injury. Neurosci Bull 2013;29:229-238.

5 Volpe JJ: Brain injury in premature infants: A complex amalgam of destructive and developmental disturbances. Lancet Neurol 2009;8:110-124.

6 Chen HJ, Wei KL, Zhou CL, Yao YJ, Yang YJ, Fan XF, Gao XR, Liu XH, Qian JH, Wu BQ, Wu GQ, Zhang QM, Zhang $\mathrm{XL}$ : Incidence of brain injuries in premature infants with gestational age a parts per thousand currency sign34 weeks in ten urban hospitals in China. World J Pediatr 2013;9:17-24.

7 Rink C, Khanna S: MicroRNA in ischemic stroke etiology and pathology. Physiol Genomics 2011;43:521528.

8 Zhao F, Qu Y, Liu J, Liu H, Zhang L, Feng Y, Wang H, Gan J, Lu R, Mu D: Microarray profiling and CoExpression network analysis of LncRNAs and mRNAs in neonatal rats following hypoxic-ischemic brain damage. Sci Rep 2015;5:13850.

9 Lu D, Xu AD: Mini review: Circular RNAs as potential clinical biomarkers for disorders in the central nervous system. Fron Genet 2016;7:53.

10 Conn SJ, Pillman KA, Toubia J, Conn VM, Salmanidis M, Phillips CA, Roslan S, Schreiber AW, Gregory PA, Goodall GJ: The RNA binding protein quaking regulates formation of circRNAs. Cell 2015;160:1125-1134.

11 Lasda E, Parker R: Circular RNAs: Diversity of form and function. RNA 2014;20:1829-1842.

12 Chen I, Chen CY, Chuang TJ: Biogenesis, identifcation, and function of exonic circular RNAs. Wiley Interdiscip Rev RNA 2015;6:563-579.

13 Memczak S, Jens M, Elefsinioti A, Torti F, Krueger J, Rybak A, Maier L, Mackowiak SD, Gregersen LH, Munschauer M, Loewer A, Ziebold U, Landthaler M, Kocks C, le Noble F, Rajewsky N: Circular RNAs are a large class of animal RNAs with regulatory potency. Nature 2013;495:333-338.

14 Chen LL, Yang L: Regulation of circRNA biogenesis. RNA Biol 2015;12:381-388.

15 Suzuki H, Tsukahara T: A view of pre-mRNA splicing from RNase R resistant RNAs. Int J Mol Sci 2014;15:9331-9342.

16 Hansen TB, Jensen TI, Clausen BH, Bramsen JB, Finsen B, Damgaard CK, Kjems J: Natural RNA circles function as efficient microRNA sponges. Nature 2013;495:384-388.

-17 Westholm JO, Miura P, Olson S, Shenker S, Joseph B, Sanfilippo P, Celniker SE, Graveley BR, Lai EC: Genomewide analysis of drosophila circular RNAs reveals their structural and sequence properties and agedependent neural accumulation. Cell Rep 2014;9:1966-1980.

18 Rybak-Wolf A, Stottmeister C, Glazar P, Jens M, Pino N, Giusti S, Hanan M, Behm M, Bartok O, Ashwal-Fluss R, Herzog M, Schreyer L, Papavasileiou P, Ivanov A, Ohman M, Refojo D, Kadener S, Rajewsky N: Circular RNAs in the mammalian brain are highly abundant, conserved, and dynamically expressed. Mol Cell 2015;58:870-885.

19 Szabo L, Morey R, Palpant NJ, Wang PL, Afari N, Jiang C, Parast MM, Murry CE, Laurent LC, Salzman J: Statistically based splicing detection reveals neural enrichment and tissue-specific induction of circular RNA during human fetal development. Genome Biol 2015;16:126.

20 Gruner H, Cortes-Lopez M, Cooper DA, Bauer M, Miura P: CircRNA accumulation in the aging mouse brain. Sci Rep 2016;6:38907.

21 Chen W, Schuman E: Circular RNAs in brain and other tissues: A functional enigma. Trends Neurosci 2016;39:597-604.

22 Hansen TB, Kjems J, Damgaard CK: Circular RNA and miR-7 in cancer. Cancer Res 2013;73:5609-5612.

23 Wan L, Zhang L, Fan K, Cheng ZX, Sun QC, Wang JJ: Circular RNA-ITCH suppresses lung cancer proliferation via inhibiting the Wnt/beta-Catenin pathway. Biomed Res Int 2016;2016:1579490. 


\section{Cellular Physiology Cell Physiol Biochem 2018;49:2264-2276 \begin{tabular}{ll|l} 
and Biochemistry Published onlIne: 27 September 2018 & $\begin{array}{l}\text { (c) } 2018 \text { The Author(s). Published by S. Karger AG, Basel } \\
\text { www.karger.com/cpb }\end{array}$
\end{tabular}

24 Barbagallo D, Caponnetto A, Cirnigliaro M, Brex D, Barbagallo C, D’Angeli F, Morrone A, Caltabiano R, Barbagallo GM, Ragusa M, Di Pietro C, Hansen TB, Purrello M: CircSMARCA5 Inhibits Migration of Glioblastoma Multiforme Cells by Regulating a Molecular Axis Involving Splicing Factors SRSF1/SRSF3/ PTB. Int J Mol Sci 2018;19:E480.

25 Qian Y, Lu Y, Rui C, Qian Y, Cai M, Jia R: Potential significance of circular RNA in human placental tissue for patients with preeclampsia. Cell Physiol Biochem 2016;39:1380-1390.

-26 Boeckel JN, Jaé N, Heumüller AW, Chen W, Boon RA, Stellos K, Zeiher AM, John D, Uchida S, Dimmeler S: Identification and Characterization of Hypoxia-Regulated Endothelial Circular RNA. Circ Res 2015;117:884890.

-27 Boeckel JN, Jaé N, Heumüller AW, Chen W, Boon RA, Stellos K, Zeiher AM, John D, Uchida S, Dimmeler S: Screening circular RNA expression patterns following focal cerebral ischemia in mice. Oncotarget 2017;8:86535-86547.

28 Zhu L, Bai X, Wang S, Hu Y, Wang T, Qian L, Jiang L: Recombinant human erythropoietin augments angiogenic responses in a neonatal rat model of cerebral unilateral Hypoxia-Ischemia. Neonatology 2014;106:143-148.

29 Gao Y, Wang J, Zhao F: CIRI: An efficient and unbiased algorithm for de novo circular RNA identification. Genome Biol 2015;16:4.

30 Li H: Aligning sequence reads, clone sequences and assembly contigs with BWA-MEM. Quantitative Biology 2013.

-31 Kent WJ, Sugnet CW, Furey TS, Roskin KM, Pringle TH, Zahler AM, Haussler D: The human genome browser at UCSC. Genome Res 2002;12:996-1006.

32 Salzman J, Chen RE, Olsen MN, Wang PL, Brown PO: Cell-type specific features of circular RNA expression. Plos Genet 2013;9:e1003777.

-33 You X, Vlatkovic I, Babic A, Will T, Epstein I, Tushev G, Akbalik G, Wang M, Glock C, Quedenau C, Wang X, Hou J, Liu H, Sun W, Sambandan S, Chen T, Schuman EM, Chen W: Neural circular RNAs are derived from synaptic genes and regulated by development and plasticity. Nat Neurosci 2015;18:603-610.

-34 Liu YC, Li JR, Sun CH, Andrews E, Chao RF, Lin FM, Weng SL, Hsu SD, Huang CC, Cheng C, Liu CC, Huang HD: CircNet: A database of circular RNAs derived from transcriptome sequencing data. Nucleic Acids Res 2016;44:D209-D215.

35 Huang DW, Sherman BT, Lempicki RA: Systematic and integrative analysis of large gene lists using DAVID bioinformatics resources. Nat Protoc 2009;4:44-57.

36 Huang DW, Sherman BT, Tan Q Kir J, Liu D, Bryant D, Guo Y, Stephens R, Baseler MW, Lane HC, Lempicki RA: DAVID Bioinformatics Resources: Expanded annotation database and novel algorithms to better extract biology from large gene lists. Nucleic Acids Res 2007;35:W169-W175.

-37 Wu J, Mao X, Cai T, Luo J, Wei L: KOBAS server: A web-based platform for automated annotation and pathway identification. Nucleic Acids Res 2006;34:W720-W724.

-38 Liu Q, Zhang X, Hu X, Dai L, Fu X, Zhang J, Ao Y: Circular RNA Related to the Chondrocyte ECM Regulates MMP13 Expression by Functioning as a MiR-136 'Sponge' in Human Cartilage Degradation. Sci Rep 2016;6: 22572.

39 Liu XB, Shen Y, Pleasure DE, Deng W: The vulnerability of thalamocortical circuitry to hypoxic-ischemic injury in a mouse model of periventricular leukomalacia. BMC Neurosci 2016;17:2.

-40 Yan F, Zhang M, Meng Y, Li H, Yu L, Fu X, Tang Y, Jiang C: Erythropoietin improves hypoxic-ischemic encephalopathy in neonatal rats after short-term anoxia by enhancing angiogenesis. Brain Res 2016;1651:104-113.

41 Qu S, Yang X, Li X, Wang J, Gao Y, Shang R, Sun W, Dou K, Li H: Circular RNA: A new star of noncoding RNAs. Cancer Lett 2015;365:141-148.

42 Chen LL, Yang L: Regulation of circRNA biogenesis. Ran Biol 2015;12:381-388.

43 Jeck WR, Sorrentino JA, Wang K, Slevin MK, Burd CE, Liu J, Marzluff WF, Sharpless NE: Circular RNAs are abundant, conserved, and associated with ALU repeats. RNA 2013;19:141-157.

44 Li F, Zhang L, Li W, Deng J, Zheng J, An M, Lu J, Zhou Y: Circular RNA ITCH has inhibitory effect on ESCC by suppressing the Wnt/beta-catenin pathway. Oncotarget 2015;6:6001-6013.

45 Zhang Y, Zhang XO, Chen T, Xiang JF, Yin QF, Xing YH, Zhu S, Yang L, Chen LL: Circular intronic long noncoding RNAs. Mol Cell 2013;51:792-806. 


\section{Cellular Physiology Cell Physiol Biochem 2018;49:2264-2276

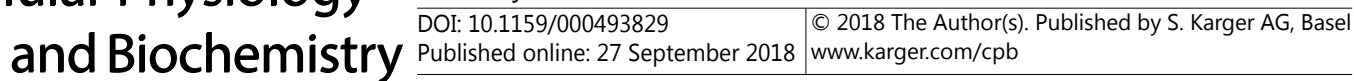 \\ Zhu et al.: Circular RNA and Periventricular White Matter Damage}

46 Li Z, Huang C, Bao C, Chen L, Lin M, Wang X, Zhong G, Yu B, Hu W, Dai L, Zhu P, Chang Z, Wu Q, Zhao Y, Jia Y, Xu P, Liu H, Shan G: Exon-intron circular RNAs regulate transcription in the nucleus. Nat Struct Mol Biol 2015;22:256-264.

47 You X, Vlatkovic I, Babic A, Will T, Epstein I, Tushev G, Akbalik G, Wang M, Glock C, Quedenau C, Wang X, Hou J, Liu H, Sun W, Sambandan S, Chen T, Schuman EM, Chen W: Neural circular RNAs are derived from synaptic genes and regulated by development and plasticity. Nat Neurosci 2015;18:603-610.

-48 Li P, Chen S, Chen H, Mo X, Li T, Shao Y, Xiao B, Guo J: Using circular RNA as a novel type of biomarker in the screening of gastric cancer. Clin Chim Acta 2015;444:132-136.

-49 Wang Z, Gerstein M, Snyder M: RNA-Seq: A revolutionary tool for transcriptomics. Nat Rev Genet 2009;10:57-63.

50 Hansen TB, Veno MT, Damgaard CK, Kjems J: Comparison of circular RNA prediction tools. Nucleic Acids Res 2016;44:e58.

-51 Jeck WR, Sharpless NE: Detecting and characterizing circular RNAs. Nat Biotechnol 2014;32:453-461.

52 Wu HJ, Zhang CY, Zhang S, Chang M, Wang HY: Microarray expression profile of circular RNAs in heart tissue of mice with myocardial Infarction-Induced heart failure. Cell Physiol Biochem 2016;39:205-216.

53 Peng L, Yuan XQ Li GC: The emerging landscape of circular RNA ciRS-7 in cancer. Oncol Rep 2015;33:26692674.

54 Fan X, Heijnen CJ, van der Kooij MA, Groenendaal F, van Bel F: The role and regulation of hypoxia-inducible factor-1alpha expression in brain development and neonatal hypoxic-ischemic brain injury. Brain Res Rev 2009;62:99-108.

55 Lu J, Jiang L, Zhu H, Zhang L, Wang T: Hypoxia-inducible factor-1alpha and erythropoietin expression in the hippocampus of neonatal rats following hypoxia-ischemia. J Nanosci Nanotechnol 2014;14:5614-5619.

56 Zheng Q, Bao C, Guo W, Li S, Chen J, Chen B, Luo Y, Lyu D, Li Y, Shi G, Liang L, Gu J, He X, Huang S: Circular RNA profiling reveals an abundant circHIPK3 that regulates cell growth by sponging multiple miRNAs. Nat Commun 2016;7:11215. 